railroad or large corporation should have a consulting neurologist, and that the chief surgeon of the road should insist that a careful neurologic examination be made of most of the injured as soon after accident as possible, especially in those cases in which there is likely to follow a suit for damages.

\section{DIAGNOSIS OF TYPHOID FEVER.}

Read at the Anniversary Meeting of the Calhoun County Medical Association at Battle Creek, Mich., Dec. 12, 1897.

\section{BY A. W. CRANE, M.D.}

KALAMAZOO, MICH.

The diagnosis of typhoid fever is a very modern achievement. A long list of diseases has been recognized by physicians from the time of Hippocrates, but the disease-entity which we call typhoid fever was not recognized as a separate disease until the early part of the nineteenth century. The name typhoid was bestowed upon it in $\$ 1829$, but even then it was confused with typhus fever, and it remained for a distinguished American physician, Gerhard of Philadelphia, finally to differentiate it from all other fevers. This was in 1837 . It was less than ten years before the discovery of anesthesia by ether. Pause and consider with what rapidity the science of clinical diag. nosis has developed. Within the memory of physicians yet living, typhoid fever, now one of the corner stones of a medical education, was for the first time differentiated as a truly distinct disease. This remarkable fact allows one to doubt the reputed powers of close and keen observation in our medical forefathers.

However, we must recognize today the great difficulty of diagnosticating the continued fevers from clinical symptoms alone. The cautious practitioner is wary of giving an early diagnosis. Long differential tables of symptorns still fail to give much certainty to his conclusions when he is called upon to decide in the beginning between such diseases as scarlatina and measles; tonsillitis and diphtheria; or simple continued fever, bilious fever, septicemia, miliary tuberculosis, malaria and typhoid fever. In the first days of almost any fever not accompanied by an eruption as in measles, or a local sign as in tonsillitis, the possibility of typhoid fever arises in the mind of the attending physician. He watches for an ascending pyrexia with morning remissions, a diarrhea, an abdominal tenderness, an enlargement of the spleen, and a rose-colored eruption over the abdomen; but he has to remember that the eruption is absent in 30 per cent. of cases and indistinct in half the remaining, that the enlargement of the spleen may be present in malaria and other diseases, that diarrhea may be absent or replaced by constipation, and that the fever curve has no constant characteristics whatever. So uncertain is the early diagnosis, that if the temperature drops on the tenth or fourteenth day the physician is often undecided in his own mind whether the patient really did have typhoid. If he has been shrewd enough not to have committed himself he is likely to call such a case bilious fever.

To illustrate the truly serious difficulty of making a differential diagnosis in some cases, at any stage, I will quote a few passages from Osler's "Practice of Medicine," second edition. He says (p. 33) "The early and intense localization of symptoms in certain organs is a frequent source of error in diagnosis. The following are the most frequent deviations from the normal course: $a$, onset with pronounced ner- vous symptoms ; $b$, with pronounced pulmonary symptoms; $c$, with intense gastro-intestinal symptoms; $d$, with symptoms of acute nephritis (p. 13). Cases coming on with severe headache, photophobia, delirium, twitching of the muscles and retraction of the the head are almost invariably regarded as cerebrospinal meningitis. I have thrice performed autopsies on cases of this kind in which no suspicion of typhoid fever had been present, the intense cerebro-spinal manifestations having dominated the scene. Fully one-half of the cases of the so-called brain fever belong to this category. Misleading pulmonary symptoms occasionally develop at the very outset of the disease. The bronchitis rarely causes error though it may be intense and attract the chief attention. More difficult are the cases setting in with a chill and followed rapidly by pneumonia. I have brought such a case before my class one week as typical pneumonia and a fortnight later shown the same case as undoubtedly one of typhoid fever. In another case in which the onset was with definite pneumonia, no spots developed; and though there were diarrhea, meteorism and the most pronounced nervous symptoms, the doubt still remained whether it was a case of typhoid fever or one of pneumonia in which severe secondary symptoms developed. There is less danger of mistaking the pneumonia which develops at the height of the disease, and yet this is possible as in a case-admitted a few years ago to my wards; a man aged 70, insensible, with a dry tongue, tremor, ecchymosis upon the wrists and ankles, no rose-spots, enlargement of the spleen, and consolidation of his right lower lobe. It was very natural, particularly since there was no history, to regard such a case as senile pneumonia with profound constitutional disturbances: but the autopsy showed the characteristic lesions of typhoid fever" (p. 33-34).

"The differential diagnosis between general miliary tuberculosis without local manifestations and typhoid fever is extremely difficult. The cough may be slight or absent. Diarrhea is rare in tuberculosis; the bowels are usually constipated, but diarrhea may occur and persist for days. In certain cases the diagnosis has been complicated still farther by the occurrence of blood in the stools. Enlargement of the spleen occurs in tuberculosis, but is neither so early nor so marked as in typhoid fever. In children, however, the enlargement may be considerable. The urine may show traces of albumin and unfortunately the diazo-reaction which is so constant in typhoid fever, is also met with in general tuberculosis" (p. 219). "Typhoid fever has been mistaken for appendicitis. I was told of a case recently, in one of the large hospitals of this country, in which the fever and the presence of a tender induration in the right iliac fossa seemed to indicate appendix disease so clearly that an operation was performed, but the induration was found to be the swollen ileum and adjacent glands. In a person who has had a previous appendicitis the diagnosis might be extremely difficult, as in a case mentioned by Da Costa. Late in the convalescence of typhoid fever the symptoms of appendicitis may develop, due to the perforation of an unhealed ulcer" (p. 440).

These extracts from Osler show us that even a master may in some cases be unable to make the diagnosis of typhoid fever, except by autopsy. In this connection it might be said that a custom of holding more frequent postmortem examinations would ele- 
vate the practice of medicine in any community. tical diagnosis of typhoid fever. Eberth's bacilli may How many atypical cases of typhoid must run their not appear in the stools until the tenth day or later. courses under other names and even end unrecog- These facts are commented upon by Osler in his secnized in death!

The means of diagnosis within the reach of the general practitioner, today, are the clinical and the laboratory methods. With the clinical we are all familiar, but the other methods have developed so rapidly, and are surrounded with so much technicality that the busy and over-worked doctor has not always taken the time to estimate them at their true value.

Much has been expected from bacteriologic examination. When any disease is proven to be caused by a particular micro-organism the problem of diagnosis would seem to be greatly simplified. The detection of the specific germ would appear to be an early and certain means of diagnosis. This is pre-eminently true of tuberculosis and diphtheria, but it is not true of typhoid fever. Yet it is sixteen years since Eberth and Koch discovered and demonstrated the etiologic relations of the typhoid bacillus-years which have formed a period of astonishing activity in bacteriologic research, so that today the technique of experimental bacteriology with its test tubes and cotton plugs, its culture media and thermostats, its guinea pigs, stains and microscopes, has become a familiar equipment in practical medicine. Nevertheless, the identification of Eberth's bacillus is too lengthy, too difficult, and too uncertain to compete with the clinical means at our disposal.

However, two methods deserve to be mentioned, namely, that of Elsner ${ }^{1}$ and that of Hiss ${ }^{2}$. The principle of Elsner's is that of almost every method yet proposed for the isolation of the typhoid bacilli. It is to add to a culture medium some substance which will prevent the growth of other bacteria without inhibiting the growth of Eberth's germ. The method of Hiss is based upon the characteristic spread of motile bacilli when grown upon acid glucose-gelatin ager kept semi-liquid at 37 degrees. Either of these methods requires two days for completion, and is too expensive to be used with any but wealthy patients, or under the auspices of a liberal board of health. As a matter of fact, it is now two years since Elsner published his method, yet we hear of no one using it in the diagnosis of typhoid fever. Within five weeks, however, the New York City Board has issued a circular announcing the new method of Dr. Hiss, its assistant bacteriologist, by which typhoid bacilli may be detected in the feces and urine of typhoid fever patients. It reads in part as follows: "The Health Department desires to thoroughly test this method and is prepared to undertake these examinations if proper specimens are furnished. The experience thus far obtained seems to indicate that the bacilli may be obtained from about 50 per cent. of all cases on the first examination and from about 90 per cent. after repeated examinations. The Health Board hopes that these examinations will prove of value not only for diagnostic purposes but also in solving sanitary questions relating to the presence in, and the time of disappearance of the bacilli from the stools during convalescence."

A prediction may be ventured that this method, like that of Elsner, will prove a failure in the prac1 For the practical details of this method see Crane, "Bacteriological
Diagnosis of Infectious Diseases." - Supplement to the Report of the
Miehtgan State Board of Health for the year 1896 , No. 463 , p. 114.
2 For a complete account of Hiss's method see Hiss, "On a method of
Isolating and Identifying Bacillus Tyhphosus."-Journal of Experimental Medicine, 1897, Vol. 2, No. 6 . ond edition. In fatal cases, even cultures from the small intestines obtained postmortem may not show the bacilli. But in the walls of the intestines, in Peyer's patches, in the ulcers, in the mesenteric glands, spleen and liver, the bacilli occur in large numbers and usually in pure culture. These facts explain the strictly limited success of the best antiseptic and eliminative forms of treatment, even including that of Dr. Woodbridge.

The obstacles to the early bacteriologic diagnosis of typhoid fever appear insurmountable. Have we then no early sign of the disease? Fourteen years ago Ehrlich tried to answer this question by an examination of the urine. He discovered the well known diazo-reaction. This valuable test has been strangely neglected by the profession as a body, and strangely misstated in some of the books, notably in the great work of Von Jaksch, in German, and in the "Medical Diagnosis" of Musser, in English. Osler, however, gives it correctly. The pink color of the form is the distinctive feature of the diazo-reaction. It is present in nearly every case of typhoid and often by the fifth or sixth day. It usually disappears before convalescence begins, and varies in intensity with the severity of the case; hence it is a sign in prognosis. Unfortunately the diazo-reaction may occasionally be present in tuberculosis, in pneumonia and in septicemia.

Up to this point we have found no single sign or symptom, or combination of signs and symptoms which are invariably and exclusively present in typhoid fever. Our clinical anchors are the continued pyrexia, the diarrhea, the dicrotic pulse, the enlarged spleen, the roseolar eruptions and the lapse of time. The diazo-reaction gives additional security. If only these symptoms were all present in all cases we would not in years past have had to wait so often for the lapse of time to clear up the clinical picture, or finally to compromise on such a diagnosis as typho-malaria, pleuro-typhoid or typho-pneumonia. We may say that the clinical symptoms alone are usually insufficient to give us a positive diagnosis at an early stage in the disease, and moreover, are often insufficient to give a diagnosis at any stage. Even the best diagnosticians teach us how often a postmortem examination will reveal an unsuspected typhoid ulceration of the bowel.

The last eighteen months have, however, placed the diagnosis of typhoid fever upon a new foundation. Now we can detect typhoid at an early stage more rapidly and with more certainty than is the case with any other infectious fever. Still more suprising than this is the fact that we can tell whether the patient has had typhoid fever, months and sometimes years after recovery. It is done by the examination of a drop of the patient's blood dried upon a piece of paper. A drop of blood thus dried could be sent across the Atlantic and examined weeks afterward in Europe with the same precision that it could at the bedside of the patient. This is the serum-diagnosis of typhoid fever, which already, like the diphtheria antitoxin and the X-ray, has become an old story to the medical public. But since it is an old story some of you may have forgotten it, and I will risk a repetition of well-known facts.

The serum-diagnosis is based upon the discovery, 
by Pfeiffer of Berlin, of bactericidal substances in a time limit of thirty minutes, the serum. diagnosis the blood of typhoid fever patients. He found, when of typhoid fever is as reliable as the bacteriologic the serum of such blood was mixed with an active diagnosis of tuberculosis and diphtheria. bouillon culture of Eberth's bacillus in a test tube, that after a few hours in an incubator the bacilli were asglutinated in masses and had settled to the bottom of the tube, leaving the upper part of the bouillon clear. His original method, which required a considerable quantity of pure, sterile serum, was complicated and difficult. Wiclal of Paris greatly simplified the process by mixing a drop of the bouillon culture of the typhoid bacilli with a drop only, of the fresh blool serum and observing the reaction under the microscope. The active movements of the bacilli were arrested and the bodies of the germs became adherent, forming clumps which are characteristic of this reaction. Further investigation by Widal and Sicard showed that the drying of the serum did not destroy its bactericidal properties. It remained for Johnson, working independently of Widal, to demon. strate that "the fluid obtained by moistening a dried blood-drop gives the reaction in a prompt and satisfactory manner, even after it had been dried for several days." This modification brings this valuable test within the sphere of practical medicine and of municipal boards of health.

The microscopic examination of the hanging-drop preparation of Eberth's bacilli leads one to wonder why it is, that in a case of typhoid fever the patient's blood and every organ in his body are not swarming with these active, squirming, whirling, shooting germs; but when once the typhoid reaction is observed, when the active germs become quiet, when their dead and dying bodies agglutinate in clumps as though they had become partially dissolved and sticky, then we see that the patient's blood has developed a poison to these germs - a bactericidal substancewithout which every case of typhoid probably would come to a fatal termination.

So remarkable a reaction speedily became the sub. ject of very numerous experiments. It was found that, aside from blood, the reaction could be obtained in typhoid cases from blister serum, from pericardial, pleural, peritoneal and joint serum, from milk, from pus and from tears. The reaction is feeble in the juices of the spleen, kidneys and liver, while the sweat, saliva, and gastric juice are without the power. Practically we may use fresh blood, dried blood, and blister serum.

A pure culture of the typhoid bacilli is an unqualfied necessity. It may be either a bouillon or an agar culture, and the bacilli may be virulent, attenuated or dead, and still give the reaction. If a dried drop of blood is dissolved in a drop of water, or if the undiluted serum is used, a fresh culture may give the reaction, occasionally, when the subject is healthy or has some disease other than typhoid. The reaction is quantitative rather than qualitative. It was found advisable, therefore, to use an attenuated culture, which is less sensitive, or else to dilute the blood or serum solution. Johnson's method is to use an attenuated culture and the dried blood without a definite dilution. He claims to thus practically eliminate the margin of error. The New York Board of Health uses a very active, virulent culture and blister serum in the dilution of one in ten. They claim reliable results in 90 per cent. of cases. As a summary of the many careful experiments, we may say that with a dilution of the serum, one in ten, or with a properly attenuated culture, and clinician, the pathologist, the chemist, the hematologist 
and the bacteriologist have been laid under tribute. And, as the product of their labors and genius, we have some of the most remarkable methods that have ever been applied to the study of disease.

\section{REPORT OF A FEW CASES OF APPEN- DICITIS.}

Read before the Camden City Medical Society, Nov. 3, 1897.

BY O. W. BRAYMER, M.D., Рн.D.

CAMDEN, N. J.

I shall only report the histories of a few cases that have recently come under my care and not undertake to give an extensive digest of the history of appendicitis and its treatment.

Case 1.-T. R., male, aged 17 years, a farm hand, had been taken at about 8 A.M. with severe cramp-like pains in the abdomen, on the right side, low down, two days before coming to the Cooper Hospital. On the day I first saw him he was suffering great pain in the right iliac region. There was some distention of the abdomen, a history of constipation, a tendency to lie with the right thigh flexed on the abdomen, marked tenderness over the region of the appendix, together with tension of the abdominal muscles of the right side, and a mass could be outlined beneath the muscular wall in this region. There was some rise of temperature and a look of intense suffering on the patient's countenance. It was decided that the case was one of appendicitis and that operation should be done at once. This had every appearance of being a case of so-called fulmi nating appendicitis. I operated in the afternoon of the same day that the patient was admitted and as soon as the inci sion was made over the region of the appendix and the cavity of the peritoneum opened there was present a fecal odor. The appendix was found to be gangrenous, firmly adherent to the under surface of the cecum and contained several coproliths. After carefully breaking up the adhesions the appendix was amputated and the stump inverted in to the bowel. The wound in the cecum was closed by a double row of fine silk sutures the darkened spots on the bowel where the appendix had adhered, were carefully cleansed and nothing more done with them, as the discoloration seemed to be only superficial. I did what I will never do again in such a case, viz.: irrigated the cavity with 1 to 10,000 bichlorid solution and followed this with distilled water. I am now of the opinion that normal saline solution is one of the best fluids to irrigate the cavity with, following such cases. The wound in the abdominal wall was closed by one layer of sutures and a space left for a drain of iodoform gauze.

This patient suffered great pain for many days after the operation and had considerable difficulty in getting his bowels to move, notwithstanding he had taken small doses of calomel from the first, followed by the salines. There was very little discharge of pus from the wound, and after about the usual length of time, where drainage is used, the opening closed. The patient entered the hospital June 23 and was up and around his room July 13 , but still complained of some pain in the region of the pubes. He was allowed to go home about one week later, and at that time was in very good condition. He was sent back to the hospital in a few weeks, suffering with intestinal obstruction, having eaten a large quantity of summer squash.

An operation by one of my colleagues revealed a constricted strangulated bowel, with much gangrene, and through the softened tissues a vast number of gquash seeds were pointing in various directions. The patient died. The obstruction which caused his death was partially due to the adhesions which had formed during the attack of appendicitis, yet the final result might perhaps have been avoided, had the patient been more careful of his diet.

Case 2.-A. B., male, aged 43 years, applied to me in March to get relief from an attack of severe abdominal pain and vomiting. He stated that he had been sick about three days, with coryza and la grippe. I found the man in a state of collapse, with general abdominal pain and vomiting. He was given morphin with atropin and strychnin hypodermatically and after about two hours was left in a fairly comfortable condition. Divided doses of calomel were given to clean out the intestinal tract and gradually the abdominal pain settled down to a point of greatest tenderness over the region of the appendix. There was a elight rise of temperature and some chilly sensations. The calomel moved the bowels satisfactorily, but there gradually developed an area of dulness in the right iliac region. There was a mass in the region of the appendix. He was removed to the hospital and was operated on at $9: 30$ P.M., March 18, 1897.

An incision over the prominent part of the swelling revealed about eight ounces of pus, which was evacuated and the cavity irrigated with a warm saline solution. A portion of omental tissue had been walled off by the pus pocket and, to avoid infection of the general peritoneal cavity, this portion of the omentum was ligated and removed. The wound in the peri toneum was closed with catgut, the muscular walls with kangaroo tendon and the skin with silkworm gut sutures, leaving an opening for drainage, the drain being composed of a small strip of iodoform gauze. This man had an uneventful recovery and three weeks after the operation was out and soon after resumed his business.

Case 3.-W. W., male, aged 21 years, came to the hospital April 12, 1897, with the following history: About two monthe previously he began to have attacks of severe abdominal pain especially around the umbilicus and in the right iliac fossa, which pain had continued with more or less severity ever since. The bowels had been opened once or twice each day and there had been no nausea.

Examination showed great tenderness about the umbilicus. and especially over McBurney's point, and the whole right side was tense and rigid. The patient when lying down kept the thigh flexed on the abdomen. No induration or fluctuation could be detected. The patient was told that he had chronic appendicitis and he decided to have the organ removed.

I made the usual incision through the abdominal walls, and found a thick, stiff appendix, pointing toward the umbilicus, somewhat adherent at the distal extremity and containing some fecal matter. The appendix was amputated close to the cecum. The stump was inverted in to the cecum and the wound in the bowel closed by means of the Lembert suture, using two rows of sutures, made of number twelve black silk. The perito neum, muscles and fascia were closed with kangaroo tendon, interrupted sutures, and the skin by silkworm gut. The patient had an uneventful recovery and was discharged cured May 4, 1897.

Case, 4. - C. K., male, aged 19 years, came to the Cooper Hospital May 5, 1897, to obtain relief from excruciating pain in the abdomen, centering in the region of the appendix. This pain had been so severe during the past few days that it debarred him from any labor. Four years ago and again two months ago there had been similar attacks, but less severe. This attack began May 3 , at $10 \mathrm{~A}$. M., with sharp pains in the right inguinal region. There was no diarrhea or vomiting until after taking some soup the day before entering the hospital. Exam ination showed a tense abdomen, right thigh flexed when patient reclined, anxious countenance, tenderness in the right iliac region and a slight rise of temperature.

I operated upon him May 6, 1897.' The appenảix was found to have an adherent bulbous distal extremity; the adhesions. were carefully broken up and the organ brought into view. The appendix was amputated near its colonic attachment. the stump was invaginated and the wound in the cecum closed with two rows of fine silk sutures, the peritoneum with a row of fine kangaroo tendon sutures and the muscles, fascia and aponeuroses were closed in a like manner. The skin was closed. by a continuous intercutaneous suture, and the wound was dressed absolutely dry. There was some extravasation of blood under the skin, which on May 12 was discharged through the center of the opening. This discharge bad a bad odor and at first was supposed to be fecal, but as no opening could be found through the muscular tissues, and as no further symptoms pointing to a fistula made their appearance, it was decided that the odor was due to the decomposition of the serum which had accumulated beneath the skin. The wound was cleansed and brought together by means of three silkworm gut sutures and recovery was uneventful. The patient was discharged cured June 4, 1897.

Case 5.-J. O., male, aged 15 years, came under my care as a private patient May 13,1897 , suffering from the ordinary symptoms of an acute attack of appendicitis. He was treated by absolute rest in bed, calomel in divided doses, together with salines and a liquid diet.

May 20 there was great tenderness in the right iliac fossa, temperature of $102.5 \mathrm{~F}$., rigidity of muscles of right side of abdominal wall a tendency to keep the right thigh flexed on the abdomen and a circumscribed swelling in the region of the appendix. I operated at 5:15 P. M. About eight ounces of very offensive curdy pus was liberated and the cavity irrigated with a saline solution. The incision was closed by means of five interrupted silkworm gut sutures, leaving room for a small iodoform gauze drain which was inserted. The patient was discharged June 3 , having made an uneventful recovery. 